\title{
Direct observation of Pt-terminating carbyne on graphene
}

\author{
Emi Kano a, cd*, Masaki Takeguchi a, b, c, d, Jun-ichi Fujita a , Ayako Hashimoto a, b, c, d
}

\begin{abstract}
Affiliations
${ }^{a}$ Graduate School of Pure and Applied Sciences, University of Tsukuba, Tsukuba, 305-8573, Japan.

${ }^{\mathrm{b}}$ Surface Physics and Structure Unit, National Institute for Materials Science, Tsukuba, 305-0047, Japan.

${ }^{\mathrm{c}}$ Global Research Center for Environment and Energy based on Nanomaterials Science, National Institute for

Materials Science, Tsukuba, 305-0047, Japan.

${ }^{\mathrm{d}}$ Transmission Electron Microscopy Station, National Institute for Materials Science, Tsukuba, 305-0047, Japan.

*Corresponding author: Tel: +81-29-859-3354 (ex. 6593), E-mail addresses: KANOU.Emi@nims.go.jp
\end{abstract}

\begin{abstract}
Theoretical studies have predicted that carbynes, single-atomic linear carbon chains, have unique properties that rival those of graphene and carbon nanotubes. However, there have been few experimental studies on carbynes owing to the lack of a reliable and effective means of production. Here, we report the direct observation of carbyne formation and dynamics by in situ transmission electron microscopy (TEM). Using Pt atoms on graphene, we succeeded in forming and observing carbynes reproducibly. Free carbon adatoms on graphene were trapped by Pt atoms, which served as nucleation sites for carbyne formation. Each end of the carbyne chain was eventually terminated by a Pt atom, and the Pt-terminating carbyne chains exhibited a variety of straight, curved, and ringed shapes. (1-1: we removed one sentence)
\end{abstract}




\section{Introduction}

Carbyne is one of the many allotropes of carbon. It is a one-dimensional (1D) structure, composed of a single atomic linear chain of $s p$-hybridized carbon atoms. Three-dimensional crystals of diamond and graphite have been known since ancient times, while low-dimensional allotropes have recently been discovered and have attracted much attention for their unique properties. In particular, zero-dimensional fullerenes [1], quasi-1D carbon nanotubes (CNTs) [2], and two-dimensional graphene [3] have notably unique electronic and mechanical properties. Carbynes have been predicted to have more remarkable properties than these materials: twice the tensile stiffness of graphene and CNT, a tension-induced increase in its electronic band gap, high stability at room temperature, and so on [4]. However, these properties have not yet been proven experimentally because of the difficulties encountered in production and during observation. In order to further the material research on carbynes, mass production should be a key factor. CNTs, for example, have become widely used since their mass production was made possible by chemical vapor deposition (CVD) [5-7].

Many methods for the production of carbynes have been demonstrated so far. For example, finite-length carbon chains have been chemically synthesized in solution [8,9], but it is difficult to determine their exact structures and properties only by spectroscopic analysis. Some researchers synthesized carbynes and observed their atomic structure by in situ transmission electron microscopy (TEM) [10-13]. They utilized an electron-beam irradiation technique to etch the graphene sheets or CNTs inside TEM equipment. The resulting carbynes were formed only accidentally. Furthermore, the method requires very careful control of the beam-irradiation conditions as well as patience to produce only a small number of carbynes. Hence, the method can only be used for fundamental studies concerning the atomic structure of carbyne and carbon-chain bonding, but it is not suitable for application purposes.

We have demonstrated a new approach to produce carbynes using Pt atoms on graphene. This is a reproducible method, and we can observe the atomic structure at the same time by TEM. Using independent Pt atoms is one of the keys to producing carbynes. The Pt atoms we report here are not clustered atoms but single, unattached ones. If Pt atoms form clusters that have dimensions of several nanometers, they end up as a catalyst 
for the growth of CNTs [7] instead of forming carbon chains. The precise role of Pt atoms will be discussed later. Another key is to use graphene, on which adsorbed atoms (Pt and C) can move around freely. Graphene also acts as a support membrane, an ultimate thin film for the detection of carbynes in TEM. Because carbynes cannot grow from Pt atoms on defective carbon structures such as amorphous carbon or hole defects in graphene, the carbynes and Pt atoms observed in our study were on clean monolayer graphene.

\section{Experimental}

\subsection{Sample Preparation}

Monolayer graphene membranes prepared by CVD on a Cu foil (Graphene Platform Corporation) were transferred to a porous carbon film on in situ heating chips (E-chips for Aduro ${ }^{\mathrm{TM}}$, Protochips) by following processes: First, a thin film of poly-methyl-methacrylate (PMMA) was spin-coated and cured on graphene/Cu. After the back of the $\mathrm{Cu}$ foil was rubbed with cotton to remove the extra graphene layer, $\mathrm{Cu}$ was then gently etched away using an ammonium persulfate solution $\left(\left(\mathrm{NH}_{4}\right)_{2} \mathrm{~S}_{2} \mathrm{O}_{8} ; 0.02 \mathrm{~g} \mathrm{ml}^{-1}\right.$, Sigma Aldrich). The PMMA/graphene film was then rinsed in deionized (DI) water and transferred to a concentrated $\mathrm{HCl}$ solution to remove residual contaminants. A thorough rinsing in DI water followed. Next, the PMMA/graphene film was transferred to the heating chip. After drying naturally for several hours, the film on the chip was heated at $180{ }^{\circ} \mathrm{C}$ in air for 30 min to improve the contact between PMMA/graphene and the carbon films on the chip. Finally, PMMA was dissolved in acetone for approximately 5 min.

Pt was deposited on the graphene membranes by a plasma sputtering system. Although there was a small amount of hydrocarbon contaminants on the graphene surface after the above sample preparation processes, the contaminants were easily dislodged under electron beam irradiation, and Pt atoms appeared on the clean graphene surface. We observed these free Pt atoms, which reached some stable sites on the graphene surface after wandering around for a while. 


\subsection{TEM characterization}

An aberration-corrected TEM (JEM-ARM200F, JEOL) was used for in situ observation. The microscope was operated at $80 \mathrm{kV}$ to prevent knock-on damage to the graphene sheets. TEM movies were acquired with a speed of 1 frame/sec. Since the samples drifted remarkably during heating observation, the positional shifts of samples in selected some snapshots were corrected and then they were averaged to improve signal-to-noise ratio, using a Gatan imaging software (Digital Micrograph, Gatan Inc.):

TEM image simulation was performed to determine the atomic structure using multislice software (xHREMTM, HREM Research Inc.), in where the following conditions were employed: 3 nm defocus spread, $0.3 \mathrm{mrad}$ beam convergence, $0 \mathrm{~mm}$ third order spherical aberration coefficient, $0.001 \mathrm{~mm}$ fifth order spherical aberration coefficient, and from $-5 \mathrm{~nm}$ to $2 \mathrm{~nm}$ defocus value. The chain and graphene sheet are divided into two slices.

We observed the migration of Pt and C atoms on a graphene surface at various temperatures with an in situ heating holder (Aduro ${ }^{\mathrm{TM}}$ heating holder, Protochips). This holder enabled us to observe graphene at atomic resolution during heating. Carbynes were observed even at room temperatures, but they were unstable. As the temperature increased towards $400{ }^{\circ} \mathrm{C}$, the carbynes became more stable. At temperatures above $600{ }^{\circ} \mathrm{C}$, Pt atoms moved rapidly by thermal vibration, and carbyne formation was not observed. Thus, all experimental results described here were obtained at $400{ }^{\circ} \mathrm{C}$.

\section{Results \& Discussions}

\subsection{Formation and Dynamics of Carbyne}

Figure 1 shows the formation of a carbyne chain with plural Pt atoms (we call this P-Carbyne). Three Pt atoms (shown in the yellow frame in Fig. 1a) captured some carbon atoms, resulting in the formation of a C-shape chain (Fig. 1b). The chain bent largely (both ends are in contact) and fluctuated a little but remained for 1 min $22 \mathrm{~s}$. In this situation, it is presumed that the bonds between the Pt atoms and the ends of the chain should 
be weak, otherwise, there were no chemical bonding and they were just in contact with Pt atom at the edge. Therefore, Pt atoms could move around rather freely until P-Carbyne suddenly turned into a linear-shape chain (Fig. 1c-e). Both ends of the chain were terminated by Pt atoms, and the chain remained motionless for more than 20 s. These atomic structures described above were illustrated in Figure 1f. A movie of the entire process of P-Carbyne formation is shown in Supplementary Movie S1.

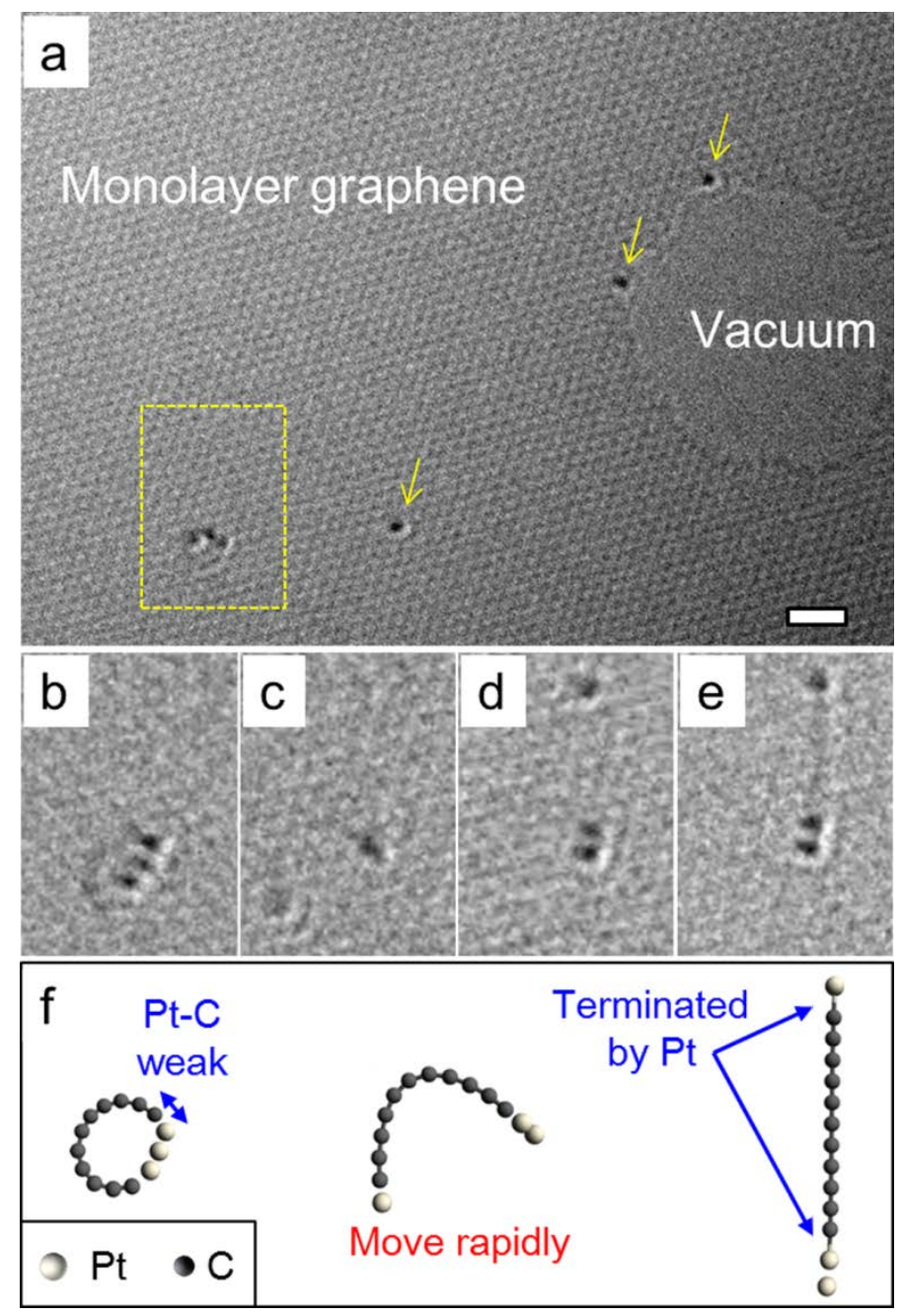

Fig. 1. Observation of carbyne with plural Pt atoms. (a) TEM image of three Pt atoms on graphene. The yellow arrows indicate single Pt atoms (atoms appear black). Scale bar is $1 \mathrm{~nm}$. (b-e) Snapshots of Supplementary Movie S1; the location is indicated by the yellow frame in (a). (b) C-shape carbyne. (c-d) Consecutive images indicating a moment of change from a C-shape to a straight structure. (e) Straight carbyne terminated by Pt atoms at both ends. (f) Schematic illustrations of the carbyne corresponding to (b-e). 
Carbon chains can be divided into four types according to their bonding structures: cumulene $(=\mathrm{C}=\mathrm{C}=)_{n}$, polyyne $(-\mathrm{C} \equiv \mathrm{C}-)_{n}$, alkane $\left(\mathrm{C}_{n} \mathrm{H}_{2 n+2}\right)$, and polyacetylene $(\mathrm{CH})_{n}$. Cumulene and polyyne are linear chains (carbynes), while alkane and polyacetylene are zigzag chains with bonding angles of $120^{\circ}$ and $109.5^{\circ}$, respectively. In the present experiment, no zigzag chain structure was observed. Therefore, we deduced that the observed structures were either cumulene or polyyne. In order to prove this, we simulated the TEM image using multislice software (Fig. 2).

Because the difference in energetic stability between cumulene and polyyne is rather small, both of the structures were theoretically predicted to co-exist under appropriate conditions. Thus, we assumed that each carbon-carbon bond had a length of 1.2-1.3 $\AA$, which falls between the bond lengths of cumulene and polyyne. Figure 2a shows a TEM image of a straight P-Carbyne after averaging 5 snapshots of the movie (one of the raw images is shown in Fig. 1e). In order to show the chain structures separately from the graphene sheet, we applied Fourier-filter to remove the underlying graphene lattice (Fig. 2b). From these experimental images, the length between the Pt atoms at both ends was measured to be approximately $1.5 \mathrm{~nm}$. On the basis of this above assumed $\mathrm{C}-\mathrm{C}$ bond length, we estimated that the overall length of $1.5 \mathrm{~nm}$ corresponds to a model composed of 11 carbons with two Pt atoms (Fig. 2c). Figure 2d shows a simulated TEM image obtained using this atomic model. We also modeled and simulated a TEM image of alkane, a hydrocarbon chain with zigzag structure (Fig. 2f, g). In Figure 2b, e, and h, the chain structures are clearly seen by applying Fourier filtering to each image (Fig. 2a, d, and g). No zigzag structure was observed in Figure 2b, and the simulated straight structures of carbynes were in good agreement with the observation. 

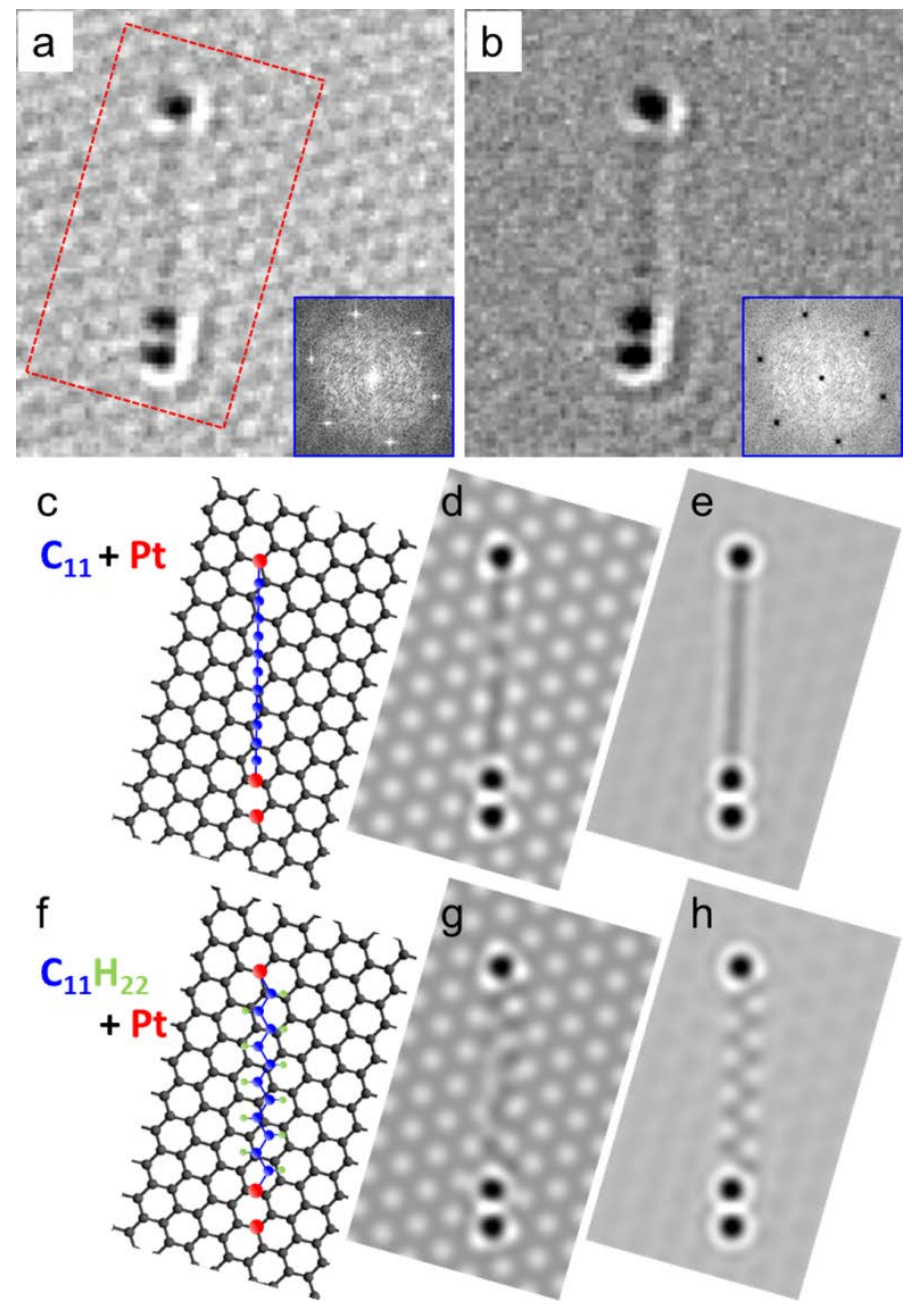

Fig. 2. Experimental and simulated images of straight carbyne (1-2, 2-1) and alkane. (a) Averaged TEM image of straight carbyne. The red frame area was modeled and simulated, as shown in (c-h). (b) Fourier filtered image of (a). The signal of graphene lattices were cut off, as shown in the inset FFT. (c-e) Modeled, simulated, and filtered image of straight carbyne $\mathrm{C}_{11}$, terminated by Pt atoms. (f-h) Modeled, simulated, and filtered image of zigzag alkane $\mathrm{C}_{11} \mathrm{H}_{22}$, terminated by Pt atoms. Simulated images ((d) and (g)) were obtained at -4 nm defocus.

After the above observation, the Pt atoms suddenly moved $3 \mathrm{~nm}$ away from the observed area. There was no hole where the Pt atoms had been before, suggesting that the source of the carbyne growth was the carbon adatoms on the graphene surface. When there were not enough carbon atoms, the Pt atoms pulled carbon atoms from the graphene lattice and created small hole defects in the structure. Under such conditions, we observed no carbynes. 
Figure 3 shows another carbyne with a single Pt atom at one end (we call this S-Carbyne). As shown in Supplementary Movie S2, the formation process of S-Carbyne was the same as that of P-Carbyne in Fig. 1: some carbon atoms were first connected to form a carbon ring with Pt atom. It is presumed that one end of the chain was in contact with a Pt atom but not chemically bonded. Thus, it soon departed from the Pt atom and was trapped by one of the defects in graphene (indicated by the red arrows in Fig. 3a). The S-Carbyne formed was more unstable than P-Carbyne. This end was hardly static, and the chain wiggled for a while (Fig. 3c-e). Finally, this chain end became stable and came to rest in a straight shape, but the end then moved suddenly to another defect site. The straight S-Carbyne changed from $2.15 \mathrm{~nm}$ (Fig. 3f) to $2.25 \mathrm{~nm}$ (Fig. 3g) in length and broke $3 \mathrm{~s}$ after the sudden movement.

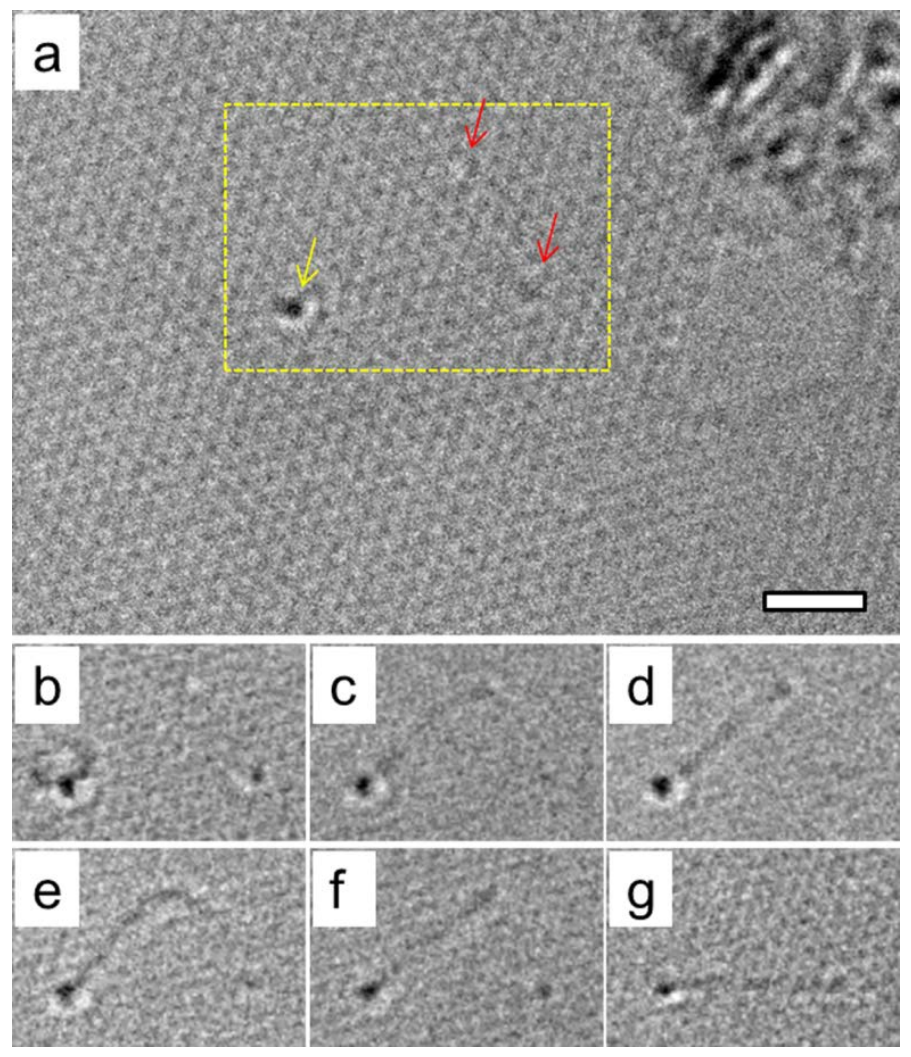

Fig. 3. Observation of carbyne with a single Pt atom. (a) TEM image of a Pt atom on graphene before carbyne formation. The yellow arrow indicates the single Pt atom, and the red arrows indicate defects (vacancies or adatoms). Scale bar is $1 \mathrm{~nm}$. (b-g) Snapshots of Supplementary Movie S2; the location is indicated by the 
yellow frame in (a). (b) Ring-shape carbyne. (c-e) Linear-shape carbyne, which was rather flexible and vibrated for a while. Between the images in (f) and (g), one end of a straight chain jumped to another defect.

\subsection{Bending Strength of Carbyne}

In general, a carbyne chain is considerably stable when straight. The persistence length is often used to quantify the bending stiffness of a polymer. It is defined as $\mathrm{l}_{\mathrm{p}}=\mathrm{EI} / \mathrm{k}_{\mathrm{B}} \mathrm{T}$, where $\mathrm{EI}$ is the bending stiffness, $\mathrm{k}_{\mathrm{B}}$ is the Bolzmann constant, and $\mathrm{T}$ is the temperature. The persistence length of carbyne at $300 \mathrm{~K}$ is roughly calculated to be $14 \mathrm{~nm}$ [4]. Using this value and equation, $\mathrm{l}_{\mathrm{p}}=6.24 \mathrm{~nm}$ for carbyne observed at $400{ }^{\circ} \mathrm{C}(673 \mathrm{~K})$. It means that the length of carbyne needs more than $6.24 \mathrm{~nm}$ for thermal fluctuations at $673 \mathrm{~K}$ to bend it.

However, in the present in situ observation, bending chains were also observed in the early process of carbyne formation. Hu et al. calculated the structures and properties of the bending chains using density functional theory [14]. They argued that because the strain energy of bending is much smaller than the energy required to break the chain, carbyne is a special soft material that is difficult to break by bending. The strain energy of bending is determined by the bending angles $\left(\theta_{i}\right)$ at all carbon atoms using the following equation:

$$
\mathrm{E}_{\text {strain }}=2.54 \sum_{\mathrm{i}=2}^{\mathrm{n}-1}\left(1-\cos \left(\theta_{i}\right)\right) \text {. }
$$

In order to simplify how the tortuosity of a curved line could be measured, they used the arc-chord ratio, which is the ratio of the length of a chain divided by the distance between its two ends. A larger arc-chord ratio means that the line is more curved. The arc-chord ratio that we observed was greater than the largest ratio in the model. For example, both ends of S-Carbyne observed were in contact and formed a ring shape (Fig. 3b). The chain length was approximately $1.8 \mathrm{~nm}$, corresponding to a chain consisting of 14 carbons, with one Pt atom at one end. Assuming that all of the bending angles at the carbon atoms were the same, the bending strain energy was calculated to be about $2.6 \mathrm{eV}$ from equation (1).

Belenkov et al. calculated the energy difference between closed ring and C-shape chain (ring with one broken bond) [16]. The energy changes in the range 122.04-149.80 kcal/mol (5.29-6.50 eV), which is close to the $\mathrm{C}=\mathrm{C}$ binding energy. The strain energy $2.6 \mathrm{eV}$, due to the large bending, is two times smaller than the energy 
required to break the chain. Because the strain energy of bending increases with a decrease in the number of atoms, in case of a carbon cluster with small number of atoms, straight chains might be formed predominantly. In our experiment, however, C-shape chain was also observed in the early stage of the formation. It may be because the carbon atoms were stable near Pt atoms. Carbynes are strong enough to maintain the carved structure without breaking, but the strain energy of bending caused the chain instability; for example, they fluctuated when they bend largely, and turned into a linear (straight) shape. These changes were rapid for both P-Carbyne (Fig.1) and S-Carbyne (Fig.3), while the latter was hardly static because of the lack of the Pt atom in one end.

\subsection{Role of Pt atoms}

We now discuss the role of Pt atoms. Do they act as a catalyst or only a nucleation site and termination material of the chain? When the bending strain energy increased enough to move a Pt atom at the end, all the carbyne chains we observed suddenly changed to a linear shape. When there was only one Pt atom that terminated one end, the Pt-free end was rather unstable and vibrated for a while because it continued to combine with carbon atoms around the chain. When multiple Pt atoms were present, the carbyne chain was stable, where both ends were terminated by Pt. Although the multiple Pt atoms did not always terminate both ends of a carbyne chain (for example, between Fig. 1b and 1c, one end of the carbyne was separated from the other for a while without connecting with a Pt atom), the Pt-free end did not remain unattached for a long time and was soon terminated by a Pt atom.

Once a carbyne chain is terminated by Pt atoms at both ends, it cannot combine with carbon atoms anymore. If the Pt atoms act as a catalyst, the chain's length would be changed by a mechanism similar to CNT synthesis: first, carbon dissolves into a metal when hydrocarbons come into contact with the metal nanoparticles. After reaching the carbon solubility limit in the metal, the as-dissolved carbon precipitates and crystallizes in the form of a cylindrical network [15]. However, the Pt atoms we observed here did not play such a role. Thus, the Pt atoms in our study worked as only nucleation sites but not as a catalyst. 


\section{Conclusions}

We have shown a novel, reproducible method of carbyne formation using Pt atoms on a clean graphene surface. Independent Pt atoms act as nucleation sites and serve to terminate the ends of carbynes. Graphene makes it possible for a carbyne chain to move freely and allows this movement to be observed directly at the same time. A carbyne chain bends largely because the bending strain is small enough for the structure to remain intact; it can even form a ring shape that finally turns into a linear shape because it is the most stable structure for a straight chain terminated by Pt atoms at both ends. Our observations prove the stability and bending strength of carbynes. Furthermore, this is the first observation of bottom-up formation of carbynes. In contrast to other top-down approaches (using carefully controlled tools to cut large materials), the observed carbyne chain was built up from single molecules: such a bottom-up approach can produce devices in parallel and is much cheaper than top-down approaches. (1-1: we removed one sentence)

\section{Acknowledgements}

We thank J. Nakamura for discussions on Pt/graphene structures, and the Centre of Materials Research for Low Carbon Emission for supporting TEM maintenance. A part of this work was supported by the "Nanotechnology Platform Project" sponsored by the Ministry of Education, Culture, Sports, Science and Technology (MEXT), Japan.

\section{Supplementary data}

Supplementary data associated with this article can be found in the online version. Movie S1, corresponding to Figure 1, shows the entire process of the formation of a carbyne chain with plural Pt atoms. It edited to be 8 times faster than real time. Movie S2, corresponding to Figure 3, shows the entire process of the formation of a carbyne chain with a single Pt atom. It edited to be 4 times faster than real time. Both movies are in MP4 format. 


\section{References and Notes}

[1] Kroto HW, Heath JR, O’Brien SC, Curl RF, Smalley RE. C60: Buckminsterfullerene. Nature 1985;318:1623.

[2] Iijima S. Helical microtubules of graphitic carbon. Nature 1991;354:56-8.

[3] Geim a K, Novoselov KS. The rise of graphene. Nat Mater 2007;6:183-91.

[4] Liu M, Artyukhov V, Lee H, Xu F, Yakobson B. Carbyne from first principles: chain of C atoms, a nanorod or a nanorope. ACS Nano 2013;7:10075-82.

[5] Dai H, Kong J, Zhou C, Franklin N, Tombler T, Cassell A, et al. Controlled chemical routes to nanotube architectures, physics, and devices. J Phys Chem B 1999;103:11246-55.

[6] Hata K, Futaba DN, Mizuno K, Namai T, Yumura M, Iijima S. Water-assisted highly efficient synthesis of impurity-free single-walled carbon nanotubes. Science 2004;306:1362-4.

[7] Yuan D, Ding L, Chu H, Feng Y, McNicholas TP, Liu J. Horizontally aligned single-walled carbon nanotube on quartz from a large variety of metal catalysts. Nano Lett 2008;8:2576-9.

[8] Casari C, Li Bassi a., Ravagnan L, Siviero F, Lenardi C, Piseri P, et al. Chemical and thermal stability of carbyne-like structures in cluster-assembled carbon films. Phys Rev B 2004;69:075422.

[9] Chalifoux WA, Tykwinski RR. Synthesis of polyynes to model the sp-carbon allotrope carbyne. Nat Chem 2010;2:967-71.

[10] Troiani HE, Miki-Yoshida M, Camacho-Bragado G a., Marques M a. L, Rubio a., Ascencio J a., et al. Direct observation of the mechanical properties of single-walled carbon nanotubes and their junctions at the atomic level. Nano Lett 2003;3:751-5.

[11] Jin C, Lan H, Peng L, Suenaga K, Iijima S. Deriving carbon atomic chains from graphene. Phys Rev Lett 2009;102:205501.

[12] Chuvilin A, Meyer JC, Algara-Siller G, Kaiser U. From graphene constrictions to single carbon chains. New J Phys 2009;11:083019.

[13] Casillas G, Mayoral A, Liu M, Ponce A, Artyukhov VI, Yakobson BI, et al. New insights into the 
properties and interactions of carbon chains as revealed by HRTEM and DFT analysis. Carbon 2014;66:436-41.

[14] Hu YH. Bending effect of sp-hybridized carbon (carbyne) chains on their structures and properties. J Phys Chem C 2011;115:1843-50.

[15] Kumar M, Carbon nanotube synthesis and growth mechanism. In Carbon Nanotubes - Synthesis, Characterization, Application (ed. Yellampalli, S.) (InTech, Rijeka, Croatia, 2011) ISBN: 978-953-307-497-9. DOI: 10.5772/19331. Available from: http://www.intechopen.com/books/carbon-nanotubes-synthesis-characterization-applications/carbon-nanotu be-synthesis-and-growth-mechanism (published online, 20 July, 2011).

[16] Belenkov EA, Shabiev FK. Structure of new carbon phases from carbyne nanorings. Crystallogr Reports 2007;52:343-8. 